\title{
The effects of the ketogenic diet on psychiatric symptomatology, weight and metabolic dysfunction in schizophrenia patients
}

\author{
Javier Gilbert-Jaramillo1*, Dario Vargas-Pico² ${ }^{2}$ Thonny Espinosa-Mendoza², Svenja Falk², Kimberly Llanos-Fernández ${ }^{2}$, Jonathan \\ Guerrero-Haro $^{1}$, Carlos Orellana-Román ${ }^{2}$, Carlos Poveda-Loor ${ }^{1}$ José Valdevila-Figueira $^{3}$ and Christopher Palmer ${ }^{4}$ \\ ${ }^{1}$ ESPOL Polytechnic University, Escuela Superior Politecnica del Litoral, Faculty of Life Sciences, Campus Gustavo Galindo Km 30.5 Via Perimetral, P.O. Box \\ 09-01-5863, Guayaquil, Ecuador, UK \\ ${ }^{2}$ Institute of Neurosciences of Guayaquil 090514, Ecuador, UK \\ ${ }^{3}$ Addictive Behavior Unit, Institute of Neurosciences of Guayaquil, UK \\ ${ }^{4}$ Department of Postgraduate and Continuing Education, McLean hospital Harvard Medical School, USA
}

\begin{abstract}
Objective: The authors aimed to test the effect of a therapeutic ketogenic diet $(\mathrm{KD})$ on the psychotic condition, body composition and metabolic dysfunction in schizophrenic patients, as well as to report the compliance with the diet.

Method: Two Ecuadorian schizophrenic patients' male and female (twins) aged 22, were included in a six-week controlled-blinded pilot study under the therapeutic $\mathrm{KD}$. Compliance was determined by daily urine measurements (commercially available ketone strips). Body composition was analyzed using bio-impedance (Tanita SC-331S) and the clinical outcomes were assessed by blood, urine and electrocardiogram analysis. The psychiatry condition was evaluated by the PANSS scale and, a two weeks follow-up after intervention was conducted to evaluate patients' health condition.

Results: During intervention, after $~ 15$ days of ketosis, PANSS scores decreased in both female (97 to 91 ) and male (82 to 75 ) patients. Body fat decreased from $24.5 \%$ to $19.8 \%$ and $21.7 \%$ to $16.8 \%$, respectively. Interestingly, after the third week of the intervention, male patient' liver enzymes were downregulated to normal levels (AST=0-40 U/L \& ALT=0-41 U/L). No other significant clinical outcomes were observed during the study. Of relevance, both patients broke the KD in several occasions.

Conclusions: The present research showed that a short-time ketogenic diet $(\mathrm{KD})$ has positive effects in the psychiatric condition, metabolic dysfunction and body composition of young schizophrenia patients; suggesting the need of a clinical trial to corroborate its use as a co-treatment.
\end{abstract}

\section{Introduction}

Schizophrenia is a psychiatric disorder with a lifetime prevalence of $\sim 1$ percent and it is characterized by cognitive, positive and negative affective symptoms [1,2]. Although the etiology of schizophrenia remains unknown, recent evidence suggests both a hyper-responsive dopaminergic system in the associative striatum [3] and, mitochondrial dysfunction and energy metabolism alterations $[4,5]$.

The most commonly prescribed psychopharmacological intervention for patients with schizophrenia are atypical antipsychotics (AAP). All AAP currently in use, effectively block dopaminergic D2 receptors, thereby reducing or eliminating the positive symptoms of schizophrenia [6]. However, they pose serious adverse effects, such as disturbances of glucose and/or fatty acid metabolism and weight gain, thus, patients treated with AAPs frequently exhibit increased co-morbidities of obesity, hyperglycemia, type 2 diabetes mellitus and dyslipidemia [7-9].

The high fat, low carbohydrate ketogenic diet (KD) was developed as an effective non-pharmacological treatment for epileptic seizures in the 1920s [10-13], and more recently is being studied in weight loss and a variety of neurological and psychiatric disorders [14,15]. The KD utilizes a high fat (75\% of daily intake, DI), extremely low carbohydrates (lower than $5 \%$ of DI) and moderate protein intake (below $20 \%$ of
DI) $[16,17]$, to promote the use of fat-derived ketone bodies (KB), i.e. acetoacetate, -hydroxybutyric acid and acetone, as a non-glucose source of energy in the brain [18].

Furthermore, a three-week $\mathrm{KD}$ conducted in schizophrenia mice $(\mathrm{C} 57 \mathrm{BL} / 6)$ not only showed significant weight loss, but also demonstrated significant improvements in the positive, negative and cognitive symptoms (measured as ataxia, social interaction, psychomotor hyperactivity, stereotyped behavior, social withdrawal, and spatial working memory) of the mice [19]. Comparable results have been described in a recent case report of two patients with schizoaffective disorders, who showed considerable weight loss and decline in positive and negative symptoms throughout a year-long, self-prescribed KD [20]. Even though these improvements reversed when the KD was disrupted, they could be recovered when ketosis was

${ }^{*}$ Correspondence to: Javier Gilbert-Jaramillo, Department of Physiology, Anatomy and Genetics; University of Oxford, Oxford OX1 3QX, E-mail: javier.gilbertjaramillo@dpag.ox.ac.uk

Key words: ketogenic diet, schizophrenia, psychiatric conditions, clinical trial, cotrearment

Received: July 10, 2018; Accepted: July 25, 2018; Published: July 31, 2018 
regained. Similarly, a 1965 pioneer KD trial, examining its effects in 10 treatment-resistant schizophrenic patients, found that symptoms significantly decreased after two weeks following a KD [21]. Although pioneering, the study neglected to measure ketone levels and druginduced adverse effects on metabolic dysfunction could not be examined, as AAPs had yet to be developed.

Thus, this underlying pilot study aimed to examine the compliance and the effects of a controlled six-weeks therapeutic 3:1 ratio KD in Ecuadorian patients with schizophrenia; a country similar to others in South American where the typical diet consists of rice, plantain, potatoes, fruits, pasta, meat, chicken, fish, and vegetable salads; and carbohydrates constitute about $60 \%$ of the DI [22].

\section{Method}

\section{Participants}

Two 22-year old opposite-sex twins with schizophrenia were recruited at the outpatient hospital unit of the Institute of Neurosciences of Guayaquil, Ecuador to participate in a six-week therapeutic 3:1 ratio ketogenic diet pilot study, from early December 2017. The patients' parents were still the primary caregivers, responsible for administering and managing disease treatment and diet.

Study inclusion criteria were set to patients with schizophrenia, aged between 18 and 30, who have been on their concurrent atypical antipsychotic intervention for longer than four months. Exclusion were: pregnancy, lactose intolerance, vegans or vegetarians, patients with established type 2 diabetes, evidence of cardiovascular disease, osteoporosis, or kidney/hepatic problems or renal insufficiencies.

The female patient was diagnosed with schizophrenia at age 14 . Her medical history showed previous pharmacological intervention with valproic acid, lorazepam, haloperidol decanoate, levomepromazine, thioridazine, carbamazepine and fluoxetine. Her daily pharmacological therapy consisted of clozapine $(300 \mathrm{mg})$, risperidone $(6 \mathrm{mg})$, clonazepam ( $3 \mathrm{mg}$ ) and biperiden $(6 \mathrm{mg})$ for the five months prior to the commencement of the study.

The male patient was diagnosed with schizophrenia at age 18 and his medical history revealed previous pharmacological treatments with risperidone, biperiden, valproic acid, fluoxetine, lorazepam, clonazepam, lamotrigine and quetiapine. In the 23 months leading up to the study, his daily pharmacological intervention consisted of levomepromazine (150 mg), quetiapine (100 mg), valproic acid (1000 $\mathrm{mg}$ ), biperiden $(6 \mathrm{mg})$ and risperidone $(4 \mathrm{mg})$.

Both patients were maintained on their current pharmacotherapeutic regimens for the duration of the study

\section{Protocol}

Both patients received a 3:1 ratio ketogenic diet plan, which was set to a daily standard of $2000 \mathrm{kcal}$ and mainly consisted of avocado, olive oil, butter, eggs, cheese, meat, spinach and broccoli. About $87 \%$ of the daily caloric intake stemmed from fat sources, with the remainder made up of protein plus carbohydrates [23]. Therefore, total net-carbohydrate intake was calculated to be less than 15 grams per day.

Clinical baseline measurements of body composition, blood haematological clinical chemistry, urine composition, electrocardiogram (ECG) and the Positive and Negative Symptom Scale (PANSS) were examined the day prior to commencing on the dietary intervention and assessed for ketone bodies (KB; in urine), serum bilirubin, haematic biometry and minerals (sodium, magnesium and potassium, which were previously suggested to be affected by KD intervention) [24-26].

All body composition measurements, blood and urine samples were obtained at a fasted state at 7.00am, while all ECGs were recorded at $8 \mathrm{am}$. Body composition (body fat, muscle mass, bone mass, body weight and height) was analyzed using a bio-impedance total body composition analyzer (Tanita SC-331S) and recorded. Both body composition and ECG examinations were conducted and interpreted by the same trained professional. PANSS interviews were conducted by the same trained professional for both patients. Environmental conditions, i.e. luminosity and noise, were kept constant. The interviewer was blind to the patients' compliance to their dietary protocols to avoid any evaluation bias.

Ketosis was determined through daily measurements of urine ketone levels at 7 a.m., as per recommendation of Urbain \& Bertz [27], with commercially available ketone strips (Healthy Wiser), as previously measured ketonuria levels of urine clinical physiochemical analysis correlated with the commercially available urine strips, confirming its correct use to determine the nutritional ketotic state [28]. All estimated $\mathrm{KB}$ values were interpreted and recorded by the patients' parents, according to the manufacturer's instructions. Additional clinical urine analysis of $\mathrm{KB}$ was conducted in weekly increments commencing after 15 days of the start of the study. Blood tests were carried out baseline, on day 19 and in the last day of the study. Body composition was measured every 15 days, and the calculated BMI was tracked throughout the study. ECG was performed at baseline and study conclusion condition. PANSS interviews took place at baseline and after day 15 of the study protocol, in weekly intervals. PANSS score was not recorded during week four of the protocol due to patient travel. 15 days post-study, when patients had resumed their regular Ecuadorian diet, blood samples, body composition and PANSS were measured and analyzed to evaluate any lasting effects of the $\mathrm{KD}$.

\section{Ethical considerations}

The research was approved by the Institute of Neurosciences of Guayaquil (INC), and National ethics approval was obtained from the Human Research Ethics Committee of the Hospital Luis Vernaza (Guayaquil, Ecuador). Both patients and their legal caregivers, i.e. the parents, were thoroughly briefed on all research objectives, study conditions, protocols, potential risks and inconveniences and informed consent was obtained from patients and parents prior to the start of the study and assent was reconfirmed every 15 days before additional blood and urine samples were obtained.

\section{Results}

\section{Diet compliance and Tolerability}

Both patients broke the ketogenic diet on several occasions. The female patient did not fully comply with the prescribed diet for the first 21 days of the study and frequently consumed sweets and fruits. Following non-compliance during the December holiday period, she fully complied with the ketogenic diet and remained in moderate/high ketosis for 15 consecutive days. The male patient fully complied with the diet for the first three weeks of the study; achieving moderate/high ketosis within 3 days of commencing the diet. He remained in ketosis for 18 consecutive days. However, following a beak with the diet during the December holiday and new year's evening celebrations, the male patient was unable to remain in moderate/high ketosis for longer than $\sim 4$ consecutive days. Moreover, both patients reported that adherence to the ketogenic dietary protocol for longer than 14 days was problematic 
due to the onset of severe, high sugar food cravings for fruit, sweets and rice. The patients showed no gastrointestinal reactions, e.g. diarrhea, vomiting and constipation in reaction to the ketogenic diet.

\section{Female patient}

At baseline, the female patient had a calculated BMI of $21.3 \mathrm{~kg} / \mathrm{m}^{2}$ and a measured body composition of $12.5 \mathrm{~kg}$ of body fat (24.5\%), 37.2 $\mathrm{kg}$ of muscle mass and $2 \mathrm{~kg}$ of bone mass. Electrocardiogram (ECG), blood and urine analyses were clinically unremarkable and the PANSS total score at baseline was 101 (positive $=28$, negative $=16$, and general psychopathology $=57$ ). Although, the patient failed to reach ketosis by day 15 of the study, her calculated BMI slightly decreased to $20.7 \mathrm{~kg} / \mathrm{m}^{2}$ and total PANSS score reduced to 97 (positive $=28$, negative $=16$, and general psychopathology $=53$ ). Body composition remained unaltered and outcome panels of blood and urine analyses remained clinically unremarkable.

At conclusion of the study, the female patient had remained in moderate/high ketosis for $\sim 15$ consecutive days and presented with a markedly decreased calculated BMI of $19.8 \mathrm{~kg} / \mathrm{m}^{2}, 9.5 \mathrm{~kg}$ of body fat $(19.8 \%)$ and $36.6 \mathrm{~kg}$ of muscle mass. There were no changes in bone mass over the course of the study. The total PANSS score decreased to 91 (positive $=26$, negative $=15$, and general psychopathology $=50$ ) . ECG results showed no alteration during this time.

Lastly, 15 days after stopping the ketogenic diet, urine ketones were no longer detectable. Positive and negative PANSS scores increased to baseline initial scores, while her general psychopathology score decreased further to 48 . The calculated BMI $\left(19.9 \mathrm{~kg} / \mathrm{m}^{2}\right)$ and body composition measurements of body fat $(9.9 \mathrm{~kg})$ and muscle mass $(36.6 \mathrm{~kg})$ slightly increased. Overall, blood clinical analyses remained clinically unremarkable.

\section{Male patient}

At baseline, the male patient had a calculated BMI of $25.1 \mathrm{~kg} / \mathrm{m}^{2}$ and a measured body composition of $14.4 \mathrm{~kg}$ of body fat (21.7\%), $49.5 \mathrm{~kg}$ of muscle mass and $2.6 \mathrm{~kg}$ of bone mass. Blood analysis revealed elevated liver enzymes (AST=46 U/L \& ALT=63 U/L). However, all other blood, urine and ECG examinations remained clinically unremarkable. The patient's PANSS total score at baseline was 82 (positive $=19$, negative $=18$, and general psychopathology $=45$ ). At $\sim 18$ consecutive days of moderate/high ketosis, his PANSS total score decreased to 75 (positive $=16$, negative $=17$, and general psychopathology $=42$ ) and liver enzymes downregulated (AST $=29 \mathrm{U} / \mathrm{L} \& \mathrm{ALT}=45 \mathrm{U} / \mathrm{L}$ ), while all other analyses of blood and urine remained clinically unremarkable.

Despite episodes of diet non-compliance at three different occasions, i.e. the patient consumed refined sugars, his BMI markedly decreased to $22.9 \mathrm{~kg} / \mathrm{m}^{2}$, and his body fat $(10.2 \mathrm{~kg},(16.8 \%))$ and muscle mass $(48 \mathrm{~kg}$ ) also reduced. Although his PANSS total score increased to 78 (positive $=17$, negative $=17$, and general psychopathology $=44$ ), it still remained below baseline testing. Interestingly, liver enzymes normalized (AST=0-40 U/L \& ALT=0-41 U/L).

Overall, there were no changes in bone mass or ECG readings over the course of the study. 15 days after stopping the ketogenic diet, urine ketones were no longer detectable. Positive and negative PANSS scores increased to baseline initial scores, while his general psychopathology decreased further to a score of 40 . The patient's calculated BMI (23.4 $\left.\mathrm{kg} / \mathrm{m}^{2}\right)$ and body composition measurements of body fat $(10.9 \mathrm{~kg})$ and muscle mass $(51.4 \mathrm{~kg}$ ) slightly increased, but still remained below baseline measurements. Overall, liver enzymes were detected at normal levels (AST $=0-40 \mathrm{U} / \mathrm{L} \& \mathrm{ALT}=0-41 \mathrm{U} / \mathrm{L}$, while all other blood analyses remained clinically unremarkable.

\section{Conclusion}

A high-fat low-carbohydrate therapeutic 3:1 ratio $\mathrm{KD}$ diet is a dramatic variation from the typical Ecuadorian diet. However, its therapeutic potential for psychiatric disorders, such as schizophrenia merits the conduct of controlled pilot studies and clinical trials $[14,15]$. The underlying study evaluated the 3:1 ratio KD in a six-week pilot study in two schizophrenia patients.

Overall, both patients showed compliance difficulties with the KD protocol and did not achieve moderate/high ketosis for prolonged times during the study. These warrant monitoring measures to be established for any follow-up studies. Anticipatory planning of celebratory foods as part of the diet may avoid a break in a future patient cohort. Similarly, sweet cravings may be counteracted in future patient cohorts by integrating low-sugar sweet treats, made with zero-caloric sweeteners, e.g. stevia or erythritol, into the KD protocol.

\section{Clinical outcomes}

The gradual decrease in blood urea levels in both patients, which was independent of a ketotic state, is inconsistent with previous studies [29], and can potentially be explained by the patients' reduced dietary protein intake [30]. Blood creatinine are in line with previous observations [31] and remained unaffected throughout the study. As liver enzymes of the female patients showed no indication of liver abnormalities, and blood urea levels of both patients increased to baseline measurements 15 days post- $\mathrm{KD}$, the $\mathrm{KD}$ is not suspected to impair liver function. Interestingly, the male patient showed elevated liver enzymes at baseline, which could be indicative of non-alcoholic fatty liver disease (NAFLD), or toxicity from valproic acid therapy [3234]. While on the $\mathrm{KD}$, his liver enzymes normalized, and remained within normal limits 15 days post-diet. Recent research suggests a KD may improve liver enzymes and reduce triglyceride levels in hepatic tissue [35], but other research is more ambiguous [36].

Moreover, the unaltered LDL cholesterol levels are in keeping with results by Sharman et al. (31) of a six weeks KD in healthy, albeit normal weight subjects. However, the underlying study failed to detect the increased HDL cholesterol in schizophrenia patients. Despite reports that minerals (sodium, potassium, magnesium and calcium) can decrease with a KD in epileptic patients [10,37,38]; no alteration of minerals in blood or bone density was exhibited during the six weeks. However, this might be attributable to the short duration of the trial, diet breaks and/or patient age. Lastly, the invariable ECG results across the study are in accordance with findings obtained by Sharma and Gulati [39].

\section{Body composition}

Body weight, and primarily body fat, decreased over the duration of the study, which is consistent with findings in the field of KD on the whole [40-42]. Bio-impedance measurements showed a decrease in the total muscle mass; however, this was partially reconstituted after two weeks of stopping the KD. The decrease in the total muscle mass could be accounted to a depletion of the skeletal muscle glycogen store to produce lactate [42-45], in the first stage of the ketogenic diet. Thereafter, the citric acid function can be maintained via the deamination of aspartate and asparagine (46), whereby preventing skeletal tissue breakdown and ensuring the maintenance of muscle mass. Thus, it appears that a KD regimen resulted in no loss of lean muscle mass $[47,48]$. 


\section{Psychiatric symptomatology}

Our findings in the psychiatric symptomatology in both patients emulate the results in animal models of Krauter et al. (19), showing improved symptomatology after $\sim 14$ consecutive days of moderate/ high ketosis. This indicates that a short-term 3:1 ratio KD is sufficient to exert an, at least short-term, psychiatric amelioration in patients with schizophrenia. Similarly, Ari et al. [49] suggested that a ketotic state might be the cause of the symptom amelioration by potentially providing a non-glucose source of energy to a potential pathological mechanism characterized by glucose impairment and altered glucose metabolism [50-52].

There is an overall trend of decreasing PANSS scores during ketosis, which is consistent with two case studies previously reported [25]. However, by comparison the PANSS scores did not decrease to the same extent, which could potentially be accounted to the shorter overall duration that patients remained in ketosis. Moreover, the general symptomatology did not return to baseline scores after the intervention but did showed an increased trend after breaking the diet. Although the PANSS interviewer remained blinded throughput the study, post-study blinding of the examiner was compromised and could have induced potential bias into the interpretation of the final PANSS interview results.

\section{Limitations of the study}

More specialized analyses e.g. change in the brain glucose uptake, to reveal the mechanism of action of the $\mathrm{KB}$ in the patients, was not available. Blood ketone beta-hydroxybutyric acid could not be measured due to inaccessibility of laboratory protocols and devices. This was a small pilot study intended to show a proof-of-concept, safety, effects and overall practicability of the KD in schizophrenia patients. Given the small sample size, statistical significance could not be determined.

\section{Future research}

A larger sample, longer study duration is needed to determine if these results can be extrapolated to the schizophrenia patient population and to allow for statistical analyses to be conducted. Future blood clinical follow-up can be done at more prolonged intervals during a 3:1 ratio KD trial in schizophrenia patients, as no significant changes were observed during the trial. The present protocol and the standard laboratory assessment recommendations from 'The Charlie Foundation for Ketogenic Therapies' can be used as a template [23].

\section{Patient perspectives}

Both patients suggested that compliance with the diet daily was difficult due to the amount of olive oil that was included in the salads, yet, when olive oil was used for making mayonnaise, due to their culture, made the diet easy and more exciting. Also, stop eating rice, potato, plantains and specially sweets were accused to be the most difficult part. Both patient reported satiety after few days of compliance with the diet reason why they stop having dinner 1 or 2 days. With regards to the behavioral condition, female patient showed happiness accusing that the two girls that regularly told to hurt herself were gone. The male patient was feeling good when he started losing fat, recovering desire to play football at the park with his friends. Overall, patients reported an improved emotional condition and diet acceptance, despite most of the time the food traditions were difficult to overcome.

\section{References}

1. Tandon R., Keshavan MS, Nasrallah HA (2008) Schizophrenia,"just the facts" what we know in 2008. 2. Epidemiology and etiology. Schizophr res 102: 1-18. [Crossref]

2. van Os J, Kapur S (2009) Schizophrenia. Lancet 374: 635-645. [Crossref]

3. Insel TR (2010) Rethinking schizophrenia. Nature 468: 187-193. [Crossref]

4. Clay HB, Sillivan S, Konradi C (2011) Mitochondrial dysfunction and pathology in bipolar disorder and schizophrenia. Int J Dev Neurosci 29: 311-324. [Crossref]

5. Rajasekaran A, Venkatasubramanian G, Berk M, Debnath M (2015). Mitochondrial dysfunction in schizophrenia: pathways, mechanisms and implications. Neurosci Biobehav Rev 48: 10-21. [Crossref]

6. Kapur S, Remington G (2001) Dopamine D 2 receptors and their role in atypical antipsychotic action: still necessary and may even be sufficient. Biol Psychiatry 50: 873-883. [Crossref]

7. Elman I, Borsook D, Lukas SE (2006) Food intake and reward mechanisms in patients with schizophrenia: implications for metabolic disturbances and treatment with secondgeneration antipsychotic agents. Neuropsychopharmacology, 31: 2091-2120. [Crossref]

8. Kang SH, Lee JI (2015) Metabolic disturbances independent of body mass in patients with schizophrenia taking atypical antipsychotics. Psychiatry Investig 12: 242-248. [Crossref]

9. Rado J (2017) The Complex Inter-relationship between Diabetes and Schizophrenia Curr Diabetes Rev. 13: 528-532. [Crossref]

10. Sampaio LPDB (2016). Ketogenic diet for epilepsy treatment. Arq Neuro-Psiquiat 74 842-848.

11. van der Louw EJ, Williams TJ, Henry-Barron BJ, Olieman JF, Duvekot JJ, et al. (2017) Ketogenic diet therapy for epilepsy during pregnancy: A case series. Seizure 45: 198201. [Crossref]

12. Stenger E, Schaeffer M, Cances C, Motte J, Auvin S, et al. (2017) Efficacy of a ketogenic diet in resistant myoclono-astatic epilepsy: A French multicenter retrospective study. Epilepsy Res 131: 64-69. [Crossref]

13. Masino SA, Rho JM (2010) Mechanisms of ketogenic diet action. Epilepsia 51: 85-85. [Crossref]

14. Bostock EC, Kirkby KC, Taylor BV (2017) The Current Status of the Ketogenic Diet in Psychiatry. Front Psychiatry 8: 43 [Crossref]

15. Rho JM, Stafstrom CE (2012) The ketogenic diet as a treatment paradigm for diverse neurological disorders. Front Pharmacol 3: 59. [Crossref]

16. Bueno NB, de Melo IS, de Oliveira SL, da Rocha AT (2013) Very-low-carbohydrate ketogenic diet v. low-fat diet for long-term weight loss: a meta-analysis of randomised controlled trials. Br J Nutr 110: 1178-1187. [Crossref]

17. Paoli A, Rubini A, Volek JS, Grimaldi KA (2013) Beyond weight loss: a review of the therapeutic uses of very-low-carbohydrate (ketogenic) diets. Eur J Clin Nutr 67: 789-796. [Crossref]

18. Morris AA (2005) Cerebral ketone body metabolism. J Inherit Metab Dis 28: 109-121. [Crossref]

19. Kraeuter AK, Loxton H, Lima BC, Rudd D, Sarnyai Z (2015) Ketogenic diet reverses behavioral abnormalities in an acute NMDA receptor hypofunction model of schizophrenia. Schizophr Res 169: 491-493. [Crossref]

20. Palmer CM (2017) Ketogenic diet in the treatment of schizoaffective disorder: Two case studies. Schizophr Res 189: 208-209. [Crossref]

21. Pacheco A, Easterling WS, Pryer MW (1965) A pilot study of the ketogenic diet in schizophrenia. Am J Psychiatry 121: 1110-1111. [Crossref]

22. Sánchez-Llaguno SN, Neira-Mosquera JA, Pérez-Rodríguez F, Moreno Rojas R (2013) Preliminary nutritional assessment of the Ecuadorian diet based on a 24-h food recall survey in Ecuador. Nutr Hosp 28: 1646-1656 [Crossref]

23. Roehl K, Sewak SL (2017) Practice Paper of the Academy of Nutrition and Dietetics: Classic and Modified Ketogenic Diets for Treatment of Epilepsy. J Acad Nutr Diet 117: 1279-1292. [Crossref]

24. Phinney SD (2004) Ketogenic diets and physical performance. Nutr Metab (Lond) 17 2 [Crossref]

25. Dashti HM, Mathew TC, Hussein T, Asfar SK, Behbahani A, et al. (2004) Long-term effects of a ketogenic diet in obese patients. Exp Clin Cardiol 9: 200-205. [Crossref] 
26. Brands MW, Manhiani MM (2012) Sodium-retaining effect of insulin in diabetes. Am J Physiol Regul Integr Comp Physiol 303: R1101-R1109. [Crossref]

27. Urbain P, Bertz H (2016) Monitoring for compliance with a ketogenic diet: what is the best time of day to test for urinary ketosis?. Nutr Metab (Lond) 13: 77. [Crossref]

28. Ye F, Li XJ, Jiang WL, Sun HB, Liu J (2015) Efficacy of and patient compliance with a ketogenic diet in adults with intractable epilepsy: a meta-analysis. J Clin Neurol 11: 26-31. [Crossref]

29. Mettler S, Mitchell N, Tipton KD (2010) Increased protein intake reduces lean body mass loss during weight loss in athletes. Med Sci Sports Exerc 42: 326-337. [Crossref]

30. Di Iorio BR, Di Micco, L, Marzocco S, De Simone E, De Blasio A, et al. (2017) Very low-protein diet (VLPD) reduces metabolic acidosis in subjects with chronic kidney disease: The "nutritional light signal" of the renal acid load. Nutrients 9: 69. [Crossref]

31. Sharman MJ, Kraemer WJ, Love DM, Avery NG, Gómez AL, et al. (2002) A ketogenic diet favorably affects serum biomarkers for cardiovascular disease in normal-weight men. J Nutr 132: 1879-1885. [Crossref]

32. Farinelli E, Giampaoli D, Cenciarini A, Cercado E, Verrotti A (2015) Valproic acid and nonalcoholic fatty liver disease: A possible association?. World J Hepatol 7: 12511257. [Crossref]

33. Verrotti A, Agostinelli S, Parisi P, Chiarelli F, Coppola G (2011) Nonalcoholic fatty liver disease in adolescents receiving valproic acid. Epilepsy Behav 20: 382-385. [Crossref]

34. Mnif L, Sellami R, \& Masmoudi J (2016) Valproic Acid and Hepatic Steatosis: A Possible Link? About a Case Report. Psychopharmacol bull 46: 59. [Crossref]

35. Mardinoglu A, Wu H, Bjornson E, Zhang C, Hakkarainen A, et al. (2018) An Integrated Understanding of the Rapid Metabolic Benefits of a Carbohydrate-Restricted Diet on Hepatic Steatosis in Humans. Cell Metab 27: 559-571. [Crossref]

36. Haghighatdoost F, Salehi-Abargouei A, Surkan PJ, Azadbakht L (2016) The effects of low carbohydrate diets on liver function tests in nonalcoholic fatty liver disease: A systematic review and meta-analysis of clinical trials. J Res Med Sci 21: 53 [Crossref]

37. Bergqvist AC, Schall JI, Stallings VA, Zemel BS (2008) Progressive bone mineral content loss in children with intractable epilepsy treated with the ketogenic diet. Am J Clin Nutr 88: 1678-1684. [Crossref]

38. McDonald TJ, Cervenka MC (2017) Ketogenic Diets for Adults with Highly Refractory Epilepsy. Epilepsy Curr 17: 346-350. [Crossref]

39. Sharma S, Gulati S (2012) The ketogenic diet and the QT interval. J Clin Neurosci 19: 181-182. [Crossref]
40. Taus M, Fumelli D, Busni D, Borroni F, Sebastianelli S, et al. (2017) A very low calorie ketogenic diet improves weight loss and quality of life in patients with adjustable gastric banding. Ann Ital Chir 88. [Crossref]

41. Abbasi J (2018). Interest in the Ketogenic Diet Grows for Weight Loss and Type 2 Diabetes. Jama, 319: 215-217. [Crossref]

42. Nieman DC, Carlson KA, Brandstater ME, Naegele RT, Blankenship JW (1987) Running endurance in 27-h-fasted humans. J Appl Physiol 63: 2502-2509. [Crossref]

43. Vendelbo MH, Clasen BF, Treebak JT, Møller L, Krusenstjerna-Hafstrøm T, et al. (2012) Insulin resistance after a 72-h fast is associated with impaired AS160 phosphorylation and accumulation of lipid and glycogen in human skeletal muscle. $\mathrm{Am}$ J Physiol Endocrinol Metab 302: E190-E200. [Crossref]

44. Volek JS, Freidenreich DJ, Saenz C, Kunces LJ, Creighton BC, et al. (2016) Metabolic characteristics of keto-adapted ultra-endurance runners. Metabolism 65: 100-110.

45. Chang CK, Borer K, Lin PJ (2017) Low-Carbohydrate-High-Fat Diet: Can it Help Exercise Performance?. J Hum Kinet 56: 81-92. [Crossref]

46. Merra G, Miranda R, Barrucco S, Gualtieri P, Mazza M, (2016) Very-low-calorie ketogenic diet with aminoacid supplement versus very low restricted-calorie diet for preserving muscle mass during weight loss: a pilot double-blind study. Eur Rev Med Pharmacol Sci 20: 2613-2621. [Crossref]

47. Gomez-Arbelaez D, Bellido D, Castro AI, Ordoñez-Mayan L, Carreira J, et al. (2016) Body composition changes after very-low-calorie ketogenic diet in obesity evaluated by 3 standardized methods. J Clin Endocrinol Metab, 102: 488-498. [Crossref]

48. Bertoli S, Trentani C, Ferraris C, De Giorgis V, Veggiotti P, et al. (2014) Long-term effects of a ketogenic diet on body composition and bone mineralization in GLUT-1 deficiency syndrome: a case series. Nutrition 30: 726-728. [Crossref]

49. Ari C, Kovács Z, Juhasz G, Murdun C, Goldhagen CR, et al. (2017) Exogenous ketone supplements reduce anxiety-related behavior in Sprague-Dawley and Wistar Albino Glaxo/Rijswijk rats. Front Mol Neurosci 9: 137. [Crossref]

50. McDermott E, de Silva P (2005) Impaired neuronal glucose uptake in pathogenesis of schizophrenia-Can GLUT 1 and GLUT 3 deficits explain imaging, post-mortem and pharmacological findings?. Med Hypotheses 65: 1076-1081. [Crossref]

51. Dwyer DS, Bradley RJ, Kablinger AS, Freeman AM (2001) Glucose metabolism in relation to schizophrenia and antipsychotic drug treatment. Ann Clin Psychiatry 13: 103-113. [Crossref]

52. Pillinger T, Beck K, Gobjila C, Donocik JG, Jauhar S, et al. (2017) Impaired glucose homeostasis in first-episode schizophrenia: a systematic review and meta-analysis. JAMA psychiatry 74: 261-269. [Crossref]

Copyright: (C2018 Gilbert-Jaramillo J. This is an open-access article distributed under the terms of the Creative Commons Attribution License, which permits unrestricted use, distribution, and reproduction in any medium, provided the original author and source are credited. 